\title{
An outbreak caused by the SARS-CoV-2 Delta (B.1.617.2) variant in a care home after partial vaccination with a single dose of the COVID-19 vaccine Vaxzevria, London, England, April 2021
}

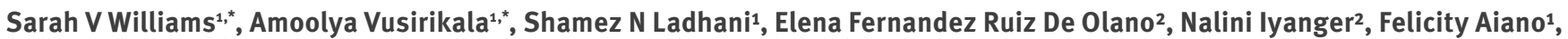
Kelly Stoker ${ }^{1}$, Guduru Gopal Rao ${ }^{3}$, Laurence John ${ }^{3}$, Bharat Patel ${ }^{1}$, Nick Andrews ${ }^{1}$, Gavin Dabrera ${ }^{1}$, Mary Ramsay ${ }^{1}, K^{2}$. Rin E Brown $^{1}$, Jamie Lopez Bernal ${ }^{1}$, Vanessa Saliba ${ }^{1}$

1. National Infection Service, Public Health England, London, United Kingdom

2. London Coronavirus Response Centre, Public Health England, London, United Kingdom

3. London North West University Healthcare NHS Trust, London, United Kingdom

* These authors contributed equally to this work and share first authorship.

Correspondence: Sarah V Williams (sarah.v.williams@phe.gov.uk)

Citation style for this article:

Williams Sarah V, Vusirikala Amoolya, Ladhani Shamez N, Fernandez Ruiz De Olano Elena, Iyanger Nalini, Aiano Felicity, Stoker Kelly, Gopal Raoz Guduru, John Laurence, Patel Bharat, Andrews Nick, Dabrera Gavin, Ramsay Mary, Brown Kevin E, Lopez Bernal Jamie, Saliba Vanessa. An outbreak caused by the SARS-CoV-2 Delta (B.1.617.2) variant in a care home after partial vaccination with a single dose of the COVID-19 vaccine Vaxzevria, London, England, April 2021. Euro Surveill. 2021;26(27):pii=2100626. https://doi.org/10.2807/1560-7917.ES.2021.26.27.2100626

We investigated a COVID-19 outbreak of the SARSCoV-2 Delta variant of concern in a London care home, where $8 / 21$ residents and $14 / 21$ staff had received a single dose of Vaxzevria (ChAdOx1-S; AstraZeneca) vaccine. We identified 24 SARS-CoV-2 infections (16 residents, 8 staff) among 40 individuals (19 residents, 21 staff); four (3 residents, 1 staff) were hospitalised, and none died. The attack rate after one vaccine dose was $35.7 \%(5 / 14)$ for staff and $81.3 \%(13 / 16)$ for residents.

In April 2021, a coronavirus disease (COVID-19) outbreak occurred at a care home in London, England, affecting both residents and staff, most of whom had received a single dose of Vaxzevria (ChAdOx1-S; AstraZeneca, Cambridge, UK) vaccine. Whole genome sequencing confirmed the outbreak was caused by the severe acute respiratory syndrome coronavirus 2 (SARS-CoV-2) Delta variant (Phylogenetic Assignment of Named Global Outbreak (Pango) lineage designation B.1.617.2). The outbreak investigation and SARS-CoV-2 serology were used to understand the impact of vaccination against infection and hospitalisation among residents and staff.

\section{Affected care home}

The care home had 21 residents (median age: 81 years; interquartile range (IQR): $78-86 ; 12$ women) and 21 permanent members of staff (median age: 49 years; IQR: $42-59 ; 16$ women). When the outbreak started, the home did not routinely employ agency staff.
Since summer 2020 , the care home conducted screening of staff with rapid lateral flow tests twice weekly and nasal-pharyngeal SARS-CoV-2 RT-PCR weekly; screening of residents with SARS-CoV-2 RT-PCR was conducted monthly $[1,2]$. If staff and residents developed COVID-19 symptoms or were hospitalised for any reason, they were also tested for SARS-CoV-2 by RT-PCR. The care home had not previously experienced a COVID-19 outbreak, defined as two or more SARSCoV-2-positive cases within a 2-week period [2].

\section{Ethical statement}

PHE has legal permission, provided by Regulation 3 of the Health Service (Control of Patient Information) Regulation 2002, to process patient confidential information for national surveillance of communicable diseases. The Investigation Protocol was reviewed and approved by the PHE Research Ethics and Governance Group (REGG) (Reference NRo252). Verbal consent for testing was obtained by care home managers from staff members and residents or their next of kin as appropriate.

\section{Outbreak evolution}

At the start of the outbreak, the index COVID-19 case was a symptomatic staff member who tested positive with a lateral flow test in early April 2021 and tested positive with RT-PCR 3 days later; the staff member had received the first Vaxzevria vaccine dose in January 2021. The staff member reported a household contact who had recently returned from India 7 days before the staff member tested positive; the household contact 


\section{FIGURE 1}

Epidemic curve of a COVID-19 outbreak caused by the SARS-CoV-2 Delta variant in care home residents and permanent members of staff, London, England, April 2021 $(\mathrm{n}=24)$

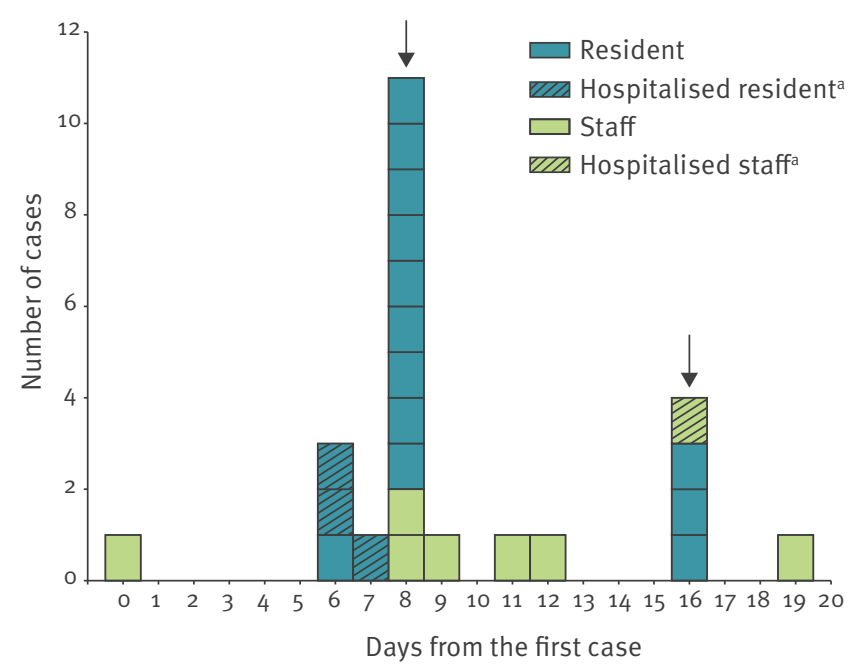

COVID-19: coronavirus disease; SARS-CoV-2: severe acute respiratory syndrome coronavirus 2 .

Arrows indicate whole care home testing for SARS-CoV-2.

a Residents and staff hospitalised with COVID-19-related symptoms. Those hospitalised because of other causes are not included.

had tested positive by RT-PCR 5 days before the staff member's positive test. An additional four COVID-19 cases at the care home were identified 6 and 7 days after the index case. These residents were admitted to the hospital, three with COVID-19 symptoms and one with an unrelated medical condition.

An outbreak was declared and, as per national guidance [2], testing of all care home residents and staff was performed twice (on day 8 and 16) after the positive result in the index case. Because of concerns about the number of cases, whole care home testing was repeated again on day 25 . In total, the whole home testing identified 15 cases, while four additional cases were detected in staff members outside whole home testing. The last case was in a staff member on day 19 , as shown in the epidemic curve (Figure 1). Among 40 swabbed individuals (19 residents, 21 staff), there were 24 cases ( 16 residents, eight staff); two residents did not consent to PCR testing. Of the cases, four were hospitalised for COVID-19 (three residents, one staff member) for 1-4 days, two required supplemental oxygen, and none required intensive care or died within 28 days of diagnosis (Table 1 ). A case was defined as a person with SARS-CoV-2 infection confirmed by RT-PCR.
TABLE 1

Characteristics of cases and non-cases in a COVID-19 outbreak caused by the SARS-CoV-2 Delta variant in a care home, London, England, April $2021(\mathrm{n}=42)$

\begin{tabular}{|c|c|c|c|c|c|c|}
\hline \multirow[t]{2}{*}{ Characteristics } & \multicolumn{2}{|c|}{$\begin{array}{l}\text { Overall } \\
(n=42)\end{array}$} & \multicolumn{2}{|c|}{$\begin{array}{l}\text { COVID-19 cases } \\
\qquad(n=24)\end{array}$} & \multicolumn{2}{|c|}{$\begin{array}{c}\text { Non-cases }^{\mathrm{a}} \\
(\mathrm{n}=18)\end{array}$} \\
\hline & $n$ & $\%$ & $n$ & $\%$ & $\mathrm{n}$ & $\%$ \\
\hline Resident & 21 & 50 & 16 & 66.7 & 5 & 27.8 \\
\hline Staff & 21 & 50 & 8 & 33.3 & 13 & 72.2 \\
\hline \multicolumn{7}{|l|}{ Sex } \\
\hline Men & 14 & 33.3 & 9 & 37.5 & 5 & 27.8 \\
\hline Women & 28 & 66.7 & 15 & 62.5 & 13 & 72.2 \\
\hline \multicolumn{7}{|l|}{ Age group (years) } \\
\hline $30-39$ & 5 & 11.9 & 2 & 8.3 & 3 & 16.7 \\
\hline $40-49$ & 6 & $14 \cdot 3$ & 2 & 8.3 & 4 & 22.2 \\
\hline $50-59$ & 6 & 14.3 & 4 & 16.7 & 2 & 11.1 \\
\hline $60-69$ & 6 & 14.3 & 2 & 8.3 & 4 & 22.2 \\
\hline $70-79$ & 5 & 11.9 & 4 & 16.7 & 1 & 5.6 \\
\hline$\geq 80$ & 14 & 33.3 & 10 & 41.7 & 4 & 22.2 \\
\hline \multicolumn{7}{|l|}{ Ethnicity } \\
\hline White & 12 & 28.6 & 10 & 41.7 & 2 & 11.1 \\
\hline Black & 2 & 4.8 & 2 & 8.3 & 0 & 0 \\
\hline Asian & 10 & 23.8 & 8 & 33.3 & 2 & 11.1 \\
\hline Mixed & 0 & 0 & 0 & 0 & 0 & 0 \\
\hline Other & 3 & 7.1 & 3 & 12.5 & 0 & 0 \\
\hline Unknown & 15 & 35.7 & 1 & 4.2 & 14 & 77.8 \\
\hline \multicolumn{7}{|l|}{ Symptomatic } \\
\hline Yes & 10 & 23.8 & 10 & 41.7 & 0 & 0 \\
\hline \multicolumn{7}{|l|}{ Hospitalised $^{b}$} \\
\hline Yes & 4 & 9.5 & 4 & 16.7 & 0 & 0 \\
\hline \multicolumn{7}{|l|}{ Vaccination status ${ }^{c}$} \\
\hline $\begin{array}{l}\text { 0-20 days post } \\
\text { dose } 1\end{array}$ & 0 & 0 & 0 & 0 & 0 & 0 \\
\hline$\geq 21$ post dose 1 & 2 & 4.9 & 2 & 8.3 & 0 & 0 \\
\hline 0-13 post dose 2 & 28 & 68.3 & 16 & 66.7 & 12 & 70.6 \\
\hline$\geq 14$ post dose 2 & 3 & 7.3 & 3 & 12.5 & 0 & 0 \\
\hline Unvaccinated & 8 & 19.5 & 3 & 12.5 & 5 & 29.4 \\
\hline
\end{tabular}

COVID-19: coronavirus disease; SARS-CoV-2: severe acute respiratory syndrome coronavirus 2 .

a Non-cases are defined as residents or permanent staff with negative SARS-CoV-2 RT-PCR tests during the outbreak period $(n=16)$ or refused a test $(n=2) . v$

b Residents and staff hospitalised with COVID-19-related symptoms. Those hospitalised because of other causes are not included.

" Vaccination with one or two doses of Vaxzevria (ChAdOx1-S; AstraZeneca, Cambridge, United Kingdom) COVID-19 vaccine $(n=41)$. One staff member received dose 1 of the Comirnaty COVID-19 (BNT162b2 mRNA, BioNTech-Pfizer, Mainz, Germany/ New York, United States) vaccine more than 21 days ago, but not included with those in the ' $\geq 21$ post dose 1 ' vaccination status category. 


\section{TABLE 2}

Attack rates in Vaxzevria-vaccinated and -unvaccinated residents and staff during a COVID-19 outbreak caused by the SARS-CoV-2 Delta variant in a care home, London, England, April $2021(\mathrm{n}=36)$

\begin{tabular}{|l|c|c|c|c|c|}
\hline \multirow{3}{*}{ Cases } & \multicolumn{4}{|c|}{ Attack rates $^{*}$} \\
\cline { 2 - 5 } & \multicolumn{2}{|c|}{ Vaccinated $^{\mathrm{a}}$} & \multicolumn{2}{|c|}{ Unvaccinated } & \multirow{2}{*}{ Reduction rate $^{\mathrm{c}}$} \\
\cline { 2 - 5 } & $\mathrm{n} / \mathrm{N}$ & $\%$ & $\mathrm{n} / \mathrm{N}$ & $\%$ & \\
\hline Staff & $5 / 14$ & 35.7 & $2 / 5$ & 40 & 10.7 \\
\hline Residents & $13 / 16$ & 81.3 & $1 / 1$ & 100 & 18.7 \\
\hline
\end{tabular}

COVID-19: coronavirus disease; SARS-CoV-2: severe acute respiratory syndrome coronavirus 2 .

a Only those who have received 1 dose or 14 days after dose 2 of Vaxzevria (ChAdOx1-S; AstraZeneca, Cambridge, United Kingdom) vaccine.

b Excludes non-cases who had antibodies from previous SARSCoV-2 infection based on serology.

Reduction rate $=(A R$ unvaccinated $-A R$ vaccinated $) / A R$ unvaccinated $\times 100$ where $A R$ is attack rate.

Residents who did not consent to PCR testing were excluded.

The outbreak was declared over in mid-May 2021, 28 days after the last case was detected. No further cases were detected following whole care home PCR testing.

\section{Vaccination status}

All residents and all staff members who were vaccinated, except one, had received the Vaxzevria vaccine. The first dose was given on 14 January 2021 (18/21 residents, $14 / 21$ staff) and the second dose was given on 1 April 2021 (18/21 residents, 13/21 staff), the same day that the index case was diagnosed with COVID-19. Exceptions include one resident who received a first dose of Vaxzevria vaccine on 12 March 2021 and one staff member who received a first dose of Comrinaty (BNT162b2 mRNA, BioNTech-Pfizer, Mainz, Germany/ New York, United States (US)) on 16 February 2021.

\section{Genomic analysis}

Whole genome sequencing was performed for RT-PCRpositive swabs that were tested at Public Health England (PHE) Colindale Virus Reference Department and had a cycle threshold (Ct) value of $<35$. Sequencing was successful for 16/24 samples: eight were confirmed and eight were probable as the Delta variant.

\section{Attack rates in Vaxzevria-vaccinated staff and residents}

The attack rate after the first vaccine dose was $35.7 \%$ $(5 / 14)$ for staff and $81.3 \%(13 / 16)$ for residents (Table 2). Because of the small size of the care home, vaccine effectiveness (VE) was not calculated. Serological testing was offered to all care home staff and residents 28 days after the last case (using the Roche Elecsys Anti-SARS-CoV-2 serology assay for the detection of anti-SARS-CoV-2 $\mathrm{N}$ antibodies and the Roche Elecsys Anti-SARS-CoV-2 S serology assay for the detection of anti-SARS-CoV-2 S antibodies, Roche Diagnostics Limited, West Sussex, United Kingdom [3,4]). Of the 24 SARS-CoV-2-positive cases, all 11 who were serologically tested had received at least one vaccine dose; all were both $\mathrm{N}$ antibody-positive (consistent with previous infection) and $\mathrm{S}$ antibody-positive (consistent with previous infection and/or vaccination). $\mathrm{S}$ antibody titres were high $(>6,000 \mathrm{AU} / \mathrm{ml})$ and consistent with a combination of vaccination and natural COVID infection.

\section{Discussion and conclusion}

More than a year into the coronavirus disease (COVID19) pandemic, new variants continue to emerge and spread rapidly across the continents. In England, the SARS-CoV-2 Delta variant, initially identified in India, was first detected in London and the North West of England in late March 2021 and declared a variant of concern on 6 May 2021 [5].

Care homes have been disproportionately affected by the pandemic, with high fatality rates reported among elderly people $[6,7]$. Care home residents and staff were, therefore, prioritised for vaccination as soon as the first COVID-19 vaccines became available [8]. Clinical trial data at the time indicated that a single dose of the Comirnaty (BNT162b2 mRNA, BioNTechPfizer, Mainz, Germany/New York, United States (US)) vaccine was estimated to provide $89 \%$ protection from symptomatic disease [9] compared with $95 \%$ for two doses given 3 weeks apart [10]. Based on these early clinical trial data indicating rapid protection after a single dose of the Comirnaty vaccine, the United Kingdom (UK) opted for an extended interval of up to 12 weeks between the two-dose schedule for COVID-19 vaccines to accelerate the rollout of the first dose of the vaccine to those at highest risk [8]. Moreover, clinical trials with the Vaxzevria vaccine demonstrated a better boost with longer intervals between vaccine doses [11]. Real-world data have demonstrated high effectiveness - especially in preventing hospitalisations and deaths - with a single dose of either vaccine, even with the more transmissible Alpha (B.1.1.7) variant [12,13] or in high risk settings such as care homes [14].

The Delta variant has emerged more recently and so there are limited data on the COVID-19 VE against this variant, especially in high risk populations. A recent preprint reported VE against symptomatic disease to be lower after one dose of either Comirnaty or Vaxzevria vaccine for Delta variant cases (33.5\%; $95 \%$ confidence interval (Cl): 20.6-44.3) compared with Alpha variant cases (51.1\%; 95\% Cl: 47.3-54.7) [15]. However, after two doses, VE increased to $87.9 \%$ (95\% $\mathrm{Cl}$ : 78.2-93.2) for Comirnaty and 59.8\% (95\% Cl: 28.9 to 77.3) for Vaxzevria [15].

The high attack rates in this care home outbreak, especially among partially vaccinated residents, is consistent with lower protection against SARS-CoV-2 infection in residents and staff who had received only one dose of the Vaxzevria vaccine within 3 months. Reassuringly, though, hospitalisation was uncommon and there were no deaths, providing some evidence that a single dose 
of vaccine may be protective against severe disease following infection with the Delta variant. Vaccination with the second dose was coincidentally arranged at the care home on the same day the index case tested positive and it is possible that this helped control the outbreak more quickly. Our findings are supported by recent research that reported VE against hospitalisation of a single dose of Vaxzevria vaccine for the Delta variant as $71 \%(95 \% \mathrm{Cl}: 51-83)$ compared with $76 \%$ (95\% Cl: 61-85) for the Alpha variant with effectiveness increasing to $92 \%(95 \% \mathrm{Cl}: 75-97)$ for the Delta variant after two doses of the vaccine [16].

There may be concern that the attack rates are based on very small numbers and so these results should not be over-interpreted as they involve a single outbreak in a small care home. It was not possible to obtain baseline serology samples on staff and residents at the start of the outbreak. In addition, serology samples at 28 days after the conclusion of the outbreak were only available on a subset of cases and non-cases. As routine testing in care homes was not available until the summer of 2020 [1], it is possible that previously asymptomatic infections during the first wave of the pandemic may have conferred some protection against reinfection for some non-cases who were reported as unvaccinated. This would lead to an under-estimation of the apparent difference the vaccine made on attack rates.

By this point, residents and permanent staff in care homes in the UK should all have been offered two vaccine doses. However, given that Delta is the dominant UK variant as of June 2021 [17], it is critical that high uptake of both doses is achieved, especially among staff, since uptake remains suboptimal in some regions [18]. Countries that have opted to extend the interval between two vaccine doses should consider offering the second dose earlier to care home residents and staff, in the context of the circulating Delta variant.

\section{Acknowledgements}

The authors would like to thank the care home staff and residents for their support in this outbreak investigation. They would also like to thank Elise Tessier, Ruth Simmons and Zahin Amin-Chowdhury at Public Health England for their help in accessing vaccination and testing data.

\section{Conflict of interest}

None declared.

\section{Authors' contributions}

Study conception (VS, JLB, KB, MR), protocol development (SW, AV, FA, KS, VS, JLB, NA, GD), data collection (SW, AV, EF, NI, FA, KS, GGR, LJ, BP), manuscript preparation (SW, AV, SL), manuscript review (all), serological assays (KB), data analysis (SW, AV, SL, NA, KB).

\section{References}

1. Dunn P, Allen L, Cameron G, Malhotra AM, Alderwick H. COVID-19 policy tracker 2020. London: The Health Foundation; 2021. Available from: https://www.health. org.uk/news-and-comment/charts-and-infographics/ covid-19-policy-tracker

2. National Health System Test and Trace. Care home COVID-19 testing guidance: for testing of staff and residents. London: United Kingdom Government; 2021. Available from: https:// assets.publishing.service.gov.uk/government/uploads/ system/uploads/attachment_data/file/988099/Care_Home Testing_Guidance_England_v12-03_rev4_DIGITAL.pdf

3. Public Health England (PHE). Evaluation of Roche Elecsys Anti-SARS-CoV-2 S serology assay for the detection of antiSARS-CoV-2 S antibodies. London: PHE; 2021. Available from: https://assets.publishing.service.gov.uk/government/uploads/ system/uploads/attachment data/file/989460/Evaluation of Roche_Elecsys_anti_SARS_CoV_2_S_assay_PHE.pdf

4. Public Health England (PHE). Evaluation of Roche Elecsys AntiSARS-CoV-2 serology assay for the detection of anti-SARSCoV-2 antibodies. London: PHE; 2020. Available from: https:// assets.publishing.service.gov.uk/government/uploads/ system/uploads/attachment data/file/891598/Evaluation of Roche Elecsys anti_SARS CoV_2 PHE 200610_v8.1_FINAL. pdf

5. The Public Health England (PHE) Variant Technical Group. SARS-COV-2 variants of concern and variants under investigation in England: Technical briefing 10. London: PHE. [Accessed: 15 Jun 2021]. Available from: https://assets. publishing.service.gov.uk/government/uploads/system/ uploads/attachment data/file/984274/Variants of Concern VOC_Technical_Briefing_10_England.pdf

6. Patel MC, Chaisson LH, Borgetti S, Burdsall D, Chugh RK, Hoff CR, et al. Asymptomatic SARS-CoV-2 infection and COVID-19 mortality during an outbreak investigation in a skilled nursing facility. Clin Infect Dis. 2020;71(11):2920-6. https://doi. org/10.1093/cid/ciaa763 PMID: 32548628

7. McMichael TM, Currie DW, Clark S, Pogosjans S, Kay M, Schwartz NG, et al. Epidemiology of Covid-19 in a longterm care facility in King County, Washington. N Engl J Med. 2020;382(21):2005-11. https://doi.org/10.1056/ NEJMoa2005412 PMID: 32220208

8. Public Health England (PHE). COVID-19: the green book, chapter 14a. London, PHE. [Accessed: 11 Jun 2021]. Available from: https://assets.publishing.service.gov.uk/government/ uploads/system/uploads/attachment_data/file/984310/ Greenbook chapter 14a_7May2021.pdf

9. United States Food and Drug Administration (FDA). PfizerBioNTech COVID-19 vaccine emergency use authorization review memorandum. Silver Spring: FDA; 2020. Available from: https://www.fda.gov/media/144416/download

10. Polack FP, Thomas SJ, Kitchin N, Absalon J, Gurtman A, Lockhart S, et al. Safety and efficacy of the BNT162b2 mRNA Covid-19 vaccine. N Engl J Med. 2020;383(27):2603-15. https:// doi.org/10.1056/NEJMoa2034577 PMID: 33301246

11. Voysey M, Costa Clemens SA, Madhi SA, Weckx LY, Folegatti PM, Aley PK, et al. Single-dose administration and the influence of the timing of the booster dose on immunogenicity and efficacy of ChAdOx1 nCoV-19 (AZD1222) vaccine: a pooled analysis of four randomised trials. Lancet. 2021:397(10277):881-91. https://doi.org/10.1016/S01406736(21)00432-3 PMID: 33617777

12. Lopez Bernal I, Andrews N, Gower C, Robertson C, Stowe Jessier $E$, et al. Effectiveness of the Pfizer-BioNTech and Oxford-AstraZeneca vaccines on covid-19 related symptoms, hospital admissions, and mortality in older adults in England: test negative case-control study. BMJ. 2021;373(1088):n1088. https://doi.org/10.1136/bmj.n1088 PMID: 33985964

13. Vasileiou ESC, Simpson CR, Robertson C, Shi T, Kerr S, Agrawal $U$, et al. Effectiveness of First Dose of COVID-19 Vaccines Against Hospital Admissions in Scotland: National Prospective Cohort Study of 5.4 Million People. 2021. https://doi.org/ DOI:10.2139/ssrn.3789264

14. Shrotri M, Krutikov M, Palmer T, Giddings R, Azmi B, Subbarao $S$, et al. Vaccine effectiveness of the first dose of ChAdOx1 $n \mathrm{CoV}-19$ and BNT162 b2 against SARS-CoV-2 infection in residents of Long-Term Care Facilities (VIVALDI study). medRxiv, 2021.03.26.21254391. https://doi.org/10.1101/2021. 03.26 .21254391

15. Bernal IL, Andrews N, Gower C, Gallagher E, Simmons R, Thelwall S, et al. Effectiveness of COVID-19 vaccines against the B.1.617.2 variant. medRxiv. 2021:2021.05.22.21257658. https://doi.org/10.1101/2021.05.22.21257658

16. Stowe J, Andrews N, Gower C, Gallagher E, Utsi L, Simmons $R$, et al. Effectiveness of COVID-19 vaccines against hospital admission with the Delta (B.1.617.2) variant. London: Public 
Health England; 2021. Pre-print. Available from: https://khub. net/web/phe-national/public-library/-/document_library/ v2WsRK3ZlEig/view/479607266

17. Public Health England (PHE). Variants: distribution of case

data, 11 June 2021. London: United Kingdom Government; 2021. Available from: https://www.gov.uk/government/publications/ covid-19-variants-genomically-confirmed-case-numbers/ variants-distribution-of-case-data-11-june-2021

18. Department of Health and Social Care. Adult social care monthly statistics, England: May 2021. London: United Kingdom Government; 2021. Available from:

https://www.gov.uk/government/statistics/adult-

social-care-in-england-monthly-statistics-may-2021/

adult-social-care-monthly-statistics-england-may-2021

\section{License, supplementary material and copyright}

This is an open-access article distributed under the terms of the Creative Commons Attribution (CC BY 4.0) Licence. You may share and adapt the material, but must give appropriate credit to the source, provide a link to the licence and indicate if changes were made.

Any supplementary material referenced in the article can be found in the online version.

This article is copyright of the authors or their affiliated institutions, 2021. 